\title{
Aportes vigorosos a la teoría de las Relaciones Internacionales
}

\section{Juan Fernando Palacio*}

\section{Reseña de libro}

Frasson-Quenoz, F. et al. (2020). Pensar, debatir y aportar a las Relaciones Internacionales.

Bogotá: Universidad Externado de Colombia. ISBN 978-958-790-432-1.

Al cumplirse un siglo de su aparición, la disciplina de las Relaciones Internacionales no para de florecer, tanto en temáticas como en perspectivas y en pertinencia, en una sociedad global cada vez más interconectada e interdependiente. Y el más reciente libro de Frasson-Quenoz y de su equipo de colegas de la Universidad Externado de Colombia y de la Pontificia Universidad Javeriana es una excelente prueba de ello.

El nuevo libro Pensar, debatir y aportar a las Relaciones Internacionales es una contribución muy significativa que demuestra que, desde Colombia, desde América Latina y desde las publicaciones de habla hispana, se está participando, con mayor rigor, de los debates más importantes que caracterizan hoy a la disciplina. Siendo esta la continuación del libro Teorias sobre Relaciones Internacionales. Perspectivas y lecturas desde América Latina (Universidad Externado de Colombia, 2018), este nuevo libro es además una corroboración del importante aporte que el Observatorio de Análisis de los Sistemas Internacionales $(\mathrm{Oa}-$ sis) le viene haciendo a la disciplina desde el contexto latinoamericano.

Circundado por secciones de presentación y de reflexiones finales muy cumplidoras que resaltan el hilo conductor y el aporte colectivo, el libro está compuesto por seis capítulos, en los que un total de siete autores participan de algunos de los debates más importantes y

* Economista y especialista en estudios políticos de la Universidad EAfIT con estudios doctorales de la Universidad de St. Gallen. Profesor de relaciones internacionales de la Universidad Pontificia Bolivariana y de comunicación gerencial de la Universidad de Antioquia. [juanf.palacio@upb.edu.co; juanfernandopalacio@gmail.com]; [https://orcid. org/0000-0002-8382-6486].

Para citar esta reseña:

Palacio, J. F. (2021). Aportes vigorosos a la teoría de las Relaciones Internacionales. Nuevos enfoques en el estudio de las sanciones internacionales [Reseña de libro: Frasson-Quenoz, F. et al. (2020). Pensar, debatiry aportar a las Relaciones Internacionales. Bogotá: Universidad Externado de Colombia. ISBN 978-958-790-432-1.]. OASIS, 34, pp. 225-228 Dor: https://doi.org/10.18601/16577558.n34.13 
apremiantes de la disciplina hoy. Estos debates son, en su orden respectivo: las tensiones entre el mainstream y las contribuciones alternativas en el contexto de la emergencia de las Relaciones Internacionales globales; el estatus de la gobernanza global como marco de referencia para la comprensión del sistema internacional; el balance del aporte de la teoría de la dependencia y la apertura hacia nuevas propuestas interdisciplinarias; los efectos de la innovación tecnológica en las reconfiguraciones futuras del sistema internacional; la búsqueda de rigor en el uso del concepto de identidad, una noción que se instaló desde hace varias décadas en el corazón de la disciplina; y, por último, pero no menos importante, la inquietud por el papel del marco institucional multilateral en el presente y futuro de la construcción y del funcionamiento del sistema internacional.

Auxiliado por los conceptos de doxa, habitus e histéresis desarrollados por Pierre Bourdieu, Florent Frasson-Quenoz hace un análisis de la redefinición de las relaciones internacionales en Relaciones Internacionales Globales, en la que se abren oportunidades para integrar, complementar y contraponer al mainstream de la disciplina, dominado por Occidente y, en especial, por Estados Unidos, otros aportes heterodoxos y alternativos surgidos, no sin dificultades, desde las márgenes, y que, aunque existen ya, no están lo suficientemente visibilizados.

Seguidamente, Erli Margarita MarínAranguren y Francisco Daniel Trejos-Mateus proponen que la gobernanza global deje de ser entendida e interpretada como un concepto y que se le reconozca la categoría de teoría en Relaciones Internacionales, lo cual puede solucionar la actual falta de visibilización de la sociedad civil global, aportando, de paso, conceptos y categorías que expliquen mejor el funcionamiento del sistema internacional.

Javier Garay, por su parte, realiza una crítica aguda a la teoría de la dependencia latinoamericana y a los modelos mentales que esta ha traído consigo en la región, proponiendo que los académicos latinoamericanos se nutran de las propuestas de otras perspectivas sugestivas, independientemente de sus procedencias geográficas, entre las cuales una muy valiosa es la Escuela Austriaca de Economía.

Manuel Alejandro Rayran-Cortés, por otro lado, se sirve del marco conceptual neogramsciano para analizar los efectos de las tecnologías de la información y las comunicaciones en lo que serán las relaciones de poder entre los actores del sistema internacional en el siglo XXI, reconociendo la disputa hegemónica en ciernes y las implicaciones para el resto de Estados.

En otra vertiente, Miguel Martínez propone que el análisis de la identidad, una noción cada vez más arraigada en las relaciones internacionales, pero que todavía es usada con algunos grados de vaguedad, se sirva de la fundamentación que en psicología se hace del concepto de identidad social, invitando así a que la disciplina se nutra de uno de los diálogos interdisciplinares que menos se han aprovechado.

Por último, Pío García exhorta a que la disciplina asuma su dimensión normativa, no solo contribuyendo a la comprensión del sistema internacional sino también iluminando a los actores hacia escenarios alternativos, en los que el multilateralismo se sigue erigiendo 
como una esperanza para afrontar problemas globales cada vez más críticos, entre los que se encuentra la supervivencia misma de la vida humana en la Tierra.

Como puede verse, pues, el libro no solo entabla diálogo y debate con algunas de las publicaciones más recientes de las áreas de especialización de cada uno de los capítulos, sino que, además, aunque varias de las contribuciones se respaldan recíprocamente, otros de los capítulos se interpelan y se contradicen. De manera especial, el libro es un buen reflejo de las grandes tensiones que existen entre, de un lado, la representatividad y la inclusividad de los aportes teóricos y, del otro, los debates sobre el valor científico de los mismos; tensiones que están presentes en la disciplina en la actualidad y que no son ajenas al resto de las ciencias sociales.

Todo esto, que no quepa duda al respecto, debe entenderse como un gran acierto de la obra, pues da cuenta, en un mismo volumen, de la diversidad de perspectivas, de enfoques, de preocupaciones y de estilos que existen en esta etapa histórica de la disciplina de las Relaciones Internacionales, que está transformándose aceleradamente, incitada, a su vez, por las nuevas corrientes de interdisciplinariedad y por las transformaciones mayores que están aconteciendo en el sistema internacional. El libro puede entenderse entonces como una constelación de visiones, a veces divergentes, pero con contrapesos y equilibrios internos que, a pesar de sus diferencias, coinciden en la meta común de buscar hacer avanzar la disciplina más allá de sus límites actuales. Esto hace que sea un libro necesario y pertinente, en el que cada capítulo es valiente y osado a su manera, consiguiendo cada uno un aporte de vanguardia.

Ahora bien, a la luz del libro, ¿¿dónde nos encontramos como disciplina?, y ¿̧hacia dónde deberíamos ir?

El libro Pensar, debatir y aportar a las Relaciones Internacionales es un gran acierto en la medida en que no se impacienta por la construcción artificiosa de una disciplina unificada, sino que entiende y promueve los aportes teóricos desde diferentes perspectivas, convirtiéndose en una plataforma en la que se acepta el diálogo entre diversos paradigmas y se valoran las contribuciones de cada uno de ellos. En efecto, mientras que una teoría unificada traería consigo el peligro del estancamiento, la coexistencia de diferentes paradigmas es una promesa inagotable de fertilidad y de avance del conocimiento, de lo cual este mismo libro constituye un buen ejemplo. Las tensiones interparadigmáticas son saludables y enriquecedoras.

La disposición al diálogo es entonces un gran primer paso, pero es solo el primero. Lo que sigue es dialogar con cada vez mayores niveles de entendimiento mutuo, y en este campo todavía tenemos como académicos varias tareas pendientes. Esto es cierto no solo en Relaciones Internacionales sino también para el tronco completo de las ciencias sociales.

Uno de los riesgos de la literatura académica especializada es el de caer en la tentación de conformarse con la validación que le dan los pares que pertenecen a su misma corriente o subespecialización. Tal validación es, por supuesto, indispensable para corroborar la construcción de conocimiento. Sin embargo, esta validación es insuficiente para 
optimizar la magnitud de su alcance. Hablarle solo a los pares de nuestra subespecialización disminuye las posibilidades de que nuestras contribuciones logren polinizar los trabajos de las demás perspectivas y paradigmas, además de que reduce el eco que nuestros trabajos puedan tener en todo el abanico de ciudadanos y actores. En otras palabras, nuestra responsabilidad social como académicos no es solo una responsabilidad lógica (entender) sino también una responsabilidad pedagógica (hacernos entender); para así entablar diálogo y fertilizar más allá de nuestra subdisciplina y hacia toda la sociedad.

En ese sentido, en dos direcciones puede seguir creciendo nuestro uso del lenguaje para potenciar las capacidades persuasivas y argumentativas de nuestras contribuciones teóricas. En primer lugar, podemos crecer aumentando la empatía hacia los aportes y las sensibilidades (tanto sociales como científicas) de las perspec- tivas que criticamos, con el fin de entablar una conversación más constructiva y fructífera con sus exponentes. En segundo lugar, podemos crecer aumentando la claridad y la sencillez en la articulación de las ideas, para así ganar certeza de que seremos comprendidos más allá de nuestros campos de subespecialización.

Lograr un equilibrio adecuado entre contribución al conocimiento y lenguaje de divulgación no es nada fácil y tal vez no exista receta; debemos aceptar procesos de ensayo y error. Pero ponernos esa meta es una tarea loable para seguir robusteciendo las futuras contribuciones a la disciplina.

El nuevo libro de Frasson-Quenoz et al. es, entonces, una excelente colección de contribuciones teóricas y una constatación de la apertura al diálogo y a la divergencia que existe entre sus autores. Estas virtudes convierten a esta obra en un paso muy importante en la dirección correcta. 